PROCEEDINGS OF THE

AMERICAN MATHEMATICAL SOCIETY

Volume 137, Number 1, January 2009, Pages 349-352

S 0002-9939(08)09517-8

Article electronically published on August 15, 2008

\title{
A LAW OF LARGE NUMBERS FOR ARITHMETIC FUNCTIONS
}

\author{
KATUSI FUKUYAMA AND YUTAKA KOMATSU
}

(Communicated by Richard C. Bradley)

\begin{abstract}
We prove the weighted strong law of large numbers for every integrable i.i.d. sequence where the weights are given by a positive strongly additive function satisfying the Lindeberg condition. This result solves one of the open problems raised in the paper by Berkes and Weber (2007).
\end{abstract}

\section{MAin RESUlt}

Let $f$ be a strongly additive arithmetic function, i.e.,

$$
\begin{aligned}
f(m n) & =f(m)+f(n) \text { if } \operatorname{gcd}(m, n)=1, \quad \text { and } \\
f\left(p^{n}\right) & =f(p) \quad \text { for all primes } p \text { and positive integers } n .
\end{aligned}
$$

Erdős-Kac [2] proved that if $f(p)=O(1)$ and $B_{p} \rightarrow \infty$ where $p$ varies along primes, then the sequence $\{f(n)\}$ obeys the central limit theorem, i.e.,

$$
\lim _{N \rightarrow \infty} \frac{1}{N} \#\left\{n \leq N \mid f(n) \leq A_{N}+x B_{N}^{1 / 2}\right\}=\frac{1}{\sqrt{2 \pi}} \int_{-\infty}^{x} e^{-u^{2} / 2} d u,
$$

where

$$
A_{n}=\sum_{p<n} \frac{f(p)}{p}, \quad B_{n}=\sum_{p<n} \frac{f^{2}(p)}{p} .
$$

Here and in the sequel, we follow the usual convention and denote the summation along the primes by $\sum_{p}$. Kubilius [4] and Shapiro [5] relaxed the condition $f(p)=$ $O(1)$ to the Lindeberg condition below:

$$
\lim _{N \rightarrow \infty} \frac{1}{B_{N}} \sum_{\left\{p<N:|f(p)| \geq \varepsilon B_{N}^{1 / 2}\right\}} \frac{f^{2}(p)}{p}=0 \text { for all } \varepsilon>0 .
$$

The purpose of this paper is to prove the following theorem and show that the irregularity of a positive strongly additive function $f$ does not have an effect on the weighted law of large numbers.

Received by the editors November 5, 2007, and, in revised form, December 17, 2007, and January 13, 2008.

2000 Mathematics Subject Classification. Primary 60F15, 11A25; Secondary 60G50.

Key words and phrases. Strong law of large numbers, strongly additive functions.

The first author was supported in part by Grant-in-Aid for Scientific Research (B) 17340029 from the Japan Society for the Promotion of Sciences.

(C)2008 American Mathematical Society Reverts to public domain 28 years from publication 
Theorem 1.1. Suppose that a positive strongly additive function $f$ satisfies the Lindeberg condition (1.1). Then for any sequence $\left\{X_{n}\right\}$ of independent and identically distributed integrable random variables, we have

$$
\lim _{N \rightarrow \infty} \sum_{n=1}^{N} f(n) X_{n} / \sum_{n=1}^{N} f(n)=E X_{1} \quad \text { a.s. }
$$

Berkes-Weber [1] proved the same conclusion by assuming

$$
f(p)=o\left(B_{p}^{1 / 2}\right) \quad \text { and } \quad B_{p} \rightarrow \infty \quad \text { as } \quad p \rightarrow \infty,
$$

which is stronger than the Lindeberg condition (1.1). They also proved it by assuming the Lindeberg condition and the following smoothness condition:

$$
\sup _{n \leq p, p^{\prime} \leq n^{2}} \frac{f(p)}{f\left(p^{\prime}\right)}=O(1)
$$

and posed the question whether the Lindeberg condition alone is sufficient to have the same conclusion. Our result, which is proved by simple calculations without using a randomization technique as is used in [1], gives an affirmative answer to this question.

\section{Proof}

We use the following asymptotics, which are proved in 1 1 under the Lindeberg condition (1.1):

$$
\begin{aligned}
& \sum_{n=1}^{N} f(n) \sim N A_{N}, \\
& \sum_{n=1}^{N} f^{2}(n) \sim N A_{N}^{2} .
\end{aligned}
$$

By (2.2), we can take a constant $C>0$ such that

$$
\sum_{n=1}^{N} f^{2}(n) \leq \frac{C N A_{N}^{2}}{2} \quad(N \geq 1)
$$
[3]:

To prove our theorem, we appeal to the characterization by Jamison-Orey-Pruitt

Lemma 2.1. Let $\left\{w_{k}\right\}$ be a sequence of positive numbers and put $W_{N}=\sum_{n=1}^{N} w_{n}$. Then

$$
\lim _{N \rightarrow \infty} \frac{1}{W_{N}} \sum_{n=1}^{N} w_{n} X_{n}=E X_{1} \quad \text { a.s. }
$$

holds for any sequence $\left\{X_{n}\right\}$ of independent and identically distributed integrable random variables if and only if

$$
\limsup _{t \rightarrow \infty} \frac{1}{t} \#\left\{n: W_{n} \leq t w_{n}\right\}<\infty
$$

We apply this characterization by putting $w_{n}=f(n)$. Because of (2.1), it is sufficient to prove

$$
\#\left\{n: n A_{n} \leq m f(n)\right\} \leq(1+C)^{2} m
$$


To begin with, we have

$$
\#\left\{n: n A_{n} \leq m f(n)\right\} \leq m+m^{2} \sum_{n>m} \frac{f^{2}(n)}{n^{2} A_{n}^{2}} .
$$

To bound the second term, we first prove

$$
\sum_{m<n \leq M} \frac{f^{2}(n)}{n^{2} A_{n}^{2}} \leq \frac{C}{m}+C \sum_{m<p<M} \frac{f(p)}{p^{2} A_{p}} \quad(m<M) .
$$

By using the partial summation method, we have

$$
\begin{aligned}
& \sum_{m<n \leq M} \frac{f^{2}(n)}{n^{2} A_{n}^{2}} \\
= & \sum_{m<n<M}\left(\sum_{k=m+1}^{n} f^{2}(k)\right)\left(\frac{1}{n^{2} A_{n}^{2}}-\frac{1}{(n+1)^{2} A_{n+1}^{2}}\right)+\left(\sum_{k=m+1}^{M} f^{2}(k)\right) \frac{1}{M^{2} A_{M}^{2}} \\
= & \sum_{m<n<M}\left(\sum_{k=m+1}^{n} f^{2}(k)\right)\left(\frac{1}{A_{n}^{2}}\left(\frac{1}{n^{2}}-\frac{1}{(n+1)^{2}}\right)+\frac{1}{(n+1)^{2}}\left(\frac{1}{A_{n}^{2}}-\frac{1}{A_{n+1}^{2}}\right)\right) \\
& +\left(\sum_{k=m+1}^{M} f^{2}(k)\right) \frac{1}{M^{2} A_{M}^{2}} .
\end{aligned}
$$

Thanks to (2.3), we have $\sum_{k=m+1}^{n} f^{2}(k) \leq C n A_{n}^{2} / 2$ and hence

$$
\begin{aligned}
\sum_{m<n \leq M} \frac{f^{2}(n)}{n^{2} A_{n}^{2}} & \leq C \sum_{m<n<M}\left(\frac{1}{n(n+1)}+\frac{A_{n+1}^{2}-A_{n}^{2}}{2 n A_{n+1}^{2}}\right)+\frac{C}{2 M} \\
& =\frac{C}{m+1}-\frac{C}{M}+C \sum_{m<n<M} \frac{A_{n+1}^{2}-A_{n}^{2}}{2 n A_{n+1}^{2}}+\frac{C}{2 M} .
\end{aligned}
$$

Since $A_{n+1}^{2}-A_{n}^{2}$ vanishes if $n$ is not prime and

$$
\frac{A_{p+1}^{2}-A_{p}^{2}}{2 p A_{p+1}^{2}}=\frac{\left(A_{p+1}+A_{p}\right)\left(A_{p+1}-A_{p}\right)}{2 p A_{p+1}^{2}} \leq \frac{2 A_{p+1} f(p)}{2 p^{2} A_{p+1}^{2}} \leq \frac{f(p)}{p^{2} A_{p}}
$$

for prime $p$, we have (2.6).

By applying (2.6), we have

$$
\begin{aligned}
\sum_{m<p<M} \frac{f(p)}{p^{2} A_{p}} & \leq \sum_{m<n \leq M} \frac{f(n)}{n^{2} A_{n}} \leq\left(\sum_{m<n \leq M} \frac{1}{n^{2}}\right)^{1 / 2}\left(\sum_{m<n \leq M} \frac{f^{2}(n)}{n^{2} A_{n}^{2}}\right)^{1 / 2} \\
& \leq \frac{1}{\sqrt{m}}\left(\frac{C}{m}+C \sum_{m<p<M} \frac{f(p)}{p^{2} A_{p}}\right)^{1 / 2} .
\end{aligned}
$$

Therefore

$$
m^{2}\left(\sum_{m<p<M} \frac{f(p)}{p^{2} A_{p}}\right)^{2} \leq C+C m \sum_{m<p<M} \frac{f(p)}{p^{2} A_{p}},
$$

and thereby

$$
\sum_{m<p<M} \frac{f(p)}{p^{2} A_{p}} \leq \frac{C m+\sqrt{C^{2} m^{2}+4 C m^{2}}}{2 m^{2}} \leq \frac{C+1}{m} .
$$


By letting $M \rightarrow \infty$, we see that (2.6) and (2.7) are valid even in the case $M=\infty$. Combining these with (2.5), we have (2.4).

\section{ACKNOWLEDGEMENTS}

The authors thank the referee and the editor for valuable comments.

\section{REFERENCES}

1. Berkes, I. and Weber, M., A law of the iterated logarithm for arithmetic functions, Proc. Amer. Math. Soc. 135 (2007) 1223-1232. MR2262929 (2007j:60043)

2. Erdös, P. and Kac, M. The Gaussian law of errors in the theory of additive number theoretic functions, Amer. J. Math. 62 (1940) 738-742. MR0002374(2:42c)

3. Jamison, B., Orey, S., and Pruitt, W., Convergence of weighted averages of independent random variables, Z. Wahrsch. verw. Geb. 4 (1965) 40-44 MR0182044 (31:6268)

4. Kubilius, J., Probabilistic methods in the theory of numbers, Uspekhi Mat. Nauk (N.S.) 11 (1956), 2(68) 31-66; Amer. Math. Soc. Translations 19 (1962) 47-85. MR0079025 (18:17d)

5. Shapiro, H. N., Distribution functions of additive arithmetic functions, Proc. Nat. Acad. Sci. USA 42 (1956) 426-430. MR0079609(18:113c)

Department of Mathematics, Kobe University, Rokko, Kobe, 657-8501 Japan

E-mail address: fukuyama@math.kobe-u.ac.jp

Graduate School of Science and Technology, Kobe University, Rokko, Kobe, 6578501 JAPAN 